\title{
The minimal flavour violating axion
}

\author{
F. Arias-Aragón and L. Merlo \\ Departamento de Física Teórica and Instituto de Física Teórica, IFT-UAM/CSIC, \\ Universidad Autónoma de Madrid, \\ Cantoblanco, 28049, Madrid, Spain \\ E-mail: fernando.arias@uam.es, luca.merlo@uam.es
}

Abstract: The solution to the Strong CP problem is analysed within the Minimal Flavour Violation (MFV) context. An Abelian factor of the complete flavour symmetry of the fermionic kinetic terms may play the role of the Peccei-Quinn symmetry in traditional axion models. Its spontaneous breaking, due to the addition of a complex scalar field to the Standard Model scalar spectrum, generates the MFV axion, which may redefine away the QCD theta parameter. It differs from the traditional QCD axion for its couplings that are governed by the fermion charges under the axial Abelian symmetry. It is also distinct from the so-called Axiflavon, as the MFV axion does not describe flavour violation, while it does induce flavour non-universality effects. The MFV axion phenomenology is discussed considering astrophysical, collider and flavour data.

Keywords: Beyond Standard Model, Global Symmetries, CP violation, Quark Masses and SM Parameters

ARXIV EPRINT: 1709.07039 


\section{Contents}

1 Introduction 1

2 The minimal flavour violation revisited 3

3 The MFV axion $\quad 6$

$\begin{array}{lll}4 & \text { Phenomenological features } & 9\end{array}$

5 Conclusions 13

$\begin{array}{lr}\text { A MFV axion transformations } & 15\end{array}$

\section{Introduction}

The search for an explanation of the fermion mass heterogeneity and of the different mixing schemes in the quark and lepton sectors underwent to a strong activity in the last 40 years. Rejecting the anthropic creed, the best strategy is to add a flavour symmetry to the Standard Model (SM) gauge group: this symmetry rules the fermion couplings, explaining the observed flavour puzzle and determining the amount of flavour violation in the theory.

The simplest possibility consists in the Abelian continuous $\mathrm{U}(1)_{\mathrm{FN}}$ flavour symmetry, dubbed Froggatt-Nielsen (FN), first considered in ref. [1]. Fermions may transform under the FN group and the Yukawa operators are invariant under $\mathrm{U}(1)_{\mathrm{FN}}$ transformations only introducing powers of an additional real scalar field $\phi$, with a non-trivial $\mathrm{U}(1)_{\mathrm{FN}}$ charge. Yukawa terms turn out to be non-renormalisable and then suppressed by suitable powers of the cut-off scale $\Lambda_{F}$ of the theory. Once the scalar field $\phi$, typically called flavon, develops a vacuum expectation value (VEV) the flavour symmetry is spontaneously broken and the Yukawa matrices can be written in terms of powers of $\langle\phi\rangle / \Lambda_{F}$. Fermion mass hierarchies and mixing angles can then be explained by an appropriate choice of the FN charges [2-7]. The large number of free parameters, one for each entry of the Yukawa matrices, has the drawback of lowering the predictive power of the model: any value of fermion masses and mixings can indeed be reproduced. Moreover, in order not to spoil the so good agreement of the SM predictions on flavour observables with the experimental data, the scale $\Lambda_{F}$ is constrained to be much larger than the electroweak (EW) scale.

More economical models in terms of number of parameters have been proposed only subsequently, based on non-Abelian discrete or continuous symmetries. The first class of theories exhibited very predictive mass textures [8-16, 16-25] and provided a certain protection from flavour violating processes [26-34]. However, the 2011 discovery of a nonvanishing, and relatively large, leptonic reactor angle [35-39] has raised strong doubts on the use of non-Abelian discrete models, whose most common prediction was a vanishing reactor angle. 
Non-Abelian continuous symmetries, instead, have shown to be effective to describe the SM flavour puzzle and to keep well under control flavour violating contributions from new physics. The most known context is the so-called Minimal Flavour Violation (MFV) that consists in the simple ansatz [40] that any source of flavour and CP violation in any theory Beyond the SM (BSM) is the one in the SM, i.e. the Yukawa couplings. This concept can be technically formulated in terms of the flavour symmetry arising in the considered Lagrangian in the limit of vanishing Yukawa couplings: the flavour group is a product of a $\mathrm{U}(3)$ factor for each field species in the given spectrum; in the SM case it is $\mathrm{U}(3)^{5}[41]{ }^{1}$ Yukawa terms are invariant under this flavour symmetry only promoting the Yukawa matrices to be fields transforming non-trivially under the non-Abelian part of $\mathrm{U}(3)^{5}$. In the original formulation [41], the Yukawa spurions are non-dynamical fields, of vanishing mass dimension, that acquire specific background values, which exactly reproduce the measured fermion masses and mixing angles. Any non-renormalisable operator constructed with the SM fields is, eventually, made flavour invariant by suitably inserting the Yukawa spurions: once expliciting the background values, the strength of the effects induced by these operators in flavour violating observables is suppressed by specific combinations of fermion masses, mixing angles and $\mathrm{CP}$ violating phases. In consequence, once considering the constraints from flavour data, the scale $\Lambda_{F}$ of the new physics originating the non-renormalisable operators, can be of the order of a few $\mathrm{TeV}$ [41-62], instead of hundreds of $\mathrm{TeV}$ in the generic case [63].

Although the MFV is a very predictive context, fermion masses and mixings are only described but not explained: no justification of the Yukawa background values is provided. Steps forward a completion of the MFV framework have been taken in refs. [64-67] (see also refs. [68-72]): the Yukawa spurions have been promoted to dynamical scalar fields and the corresponding scalar potential has been investigated. This analysis showed interesting, even if not conclusive, results: a minimum of the potential describes non-vanishing masses for the heavier charged fermions, two non-vanishing neutrino masses, almost no mixing in the quark sector, one maximal lepton mixing associated to a maximal Majorana phase, when considering the SM fermion spectrum extended by the addition of three right handed neutrinos.

Once a continuous symmetry is spontaneously broken, Goldstone Bosons (GBs) are generated. This possibility is typically avoided within the MFV context gauging (part of) the symmetry [51-55,61, 62]. On the other side, surviving GBs may represent the key ingredients to deal with other open problems in the SM. The focus in this letter will be on the Strong CP problem and it will be shown that a GB arising from the spontaneous breaking of an Abelian term of the MFV symmetry $\mathrm{U}(5)^{3}$ can be an axion.

The solution of the Strong CP problem proposed here in the MFV context follows the lines of the traditional QCD axion [73-75]: the U(1) factor that originates the MFV axion is not vectorial and it is explicitly broken by the colour anomalies; the so-called

\footnotetext{
${ }^{1}$ Once considering the BSM extension with three right-handed neutrinos, which allows for an explanation of the active neutrino masses, the flavour group is $\mathrm{U}(3)^{6}$ [42-44]. This scenario is however not predictive and a reduction of the symmetry is required. See ref. [45] for a recent update.
} 
theta-parameter,

$$
\mathscr{L}_{\mathrm{QCD}} \supset \frac{\alpha_{s}}{8 \pi} \theta_{\mathrm{QCD}} G^{a \mu \nu} \widetilde{G}_{\mu \nu}^{a}
$$

with $\widetilde{G}_{\mu \nu}^{a} \equiv \epsilon_{\mu \nu \rho \sigma} G^{a \rho \sigma}$ and $\epsilon_{\mu \nu \rho \sigma}$ the totally antisymmetric tensor such that $\epsilon_{1230}=1$, can be redefined away by a shift symmetry transformation; exactly as for the QCD axion (see for example ref. [76]), the minimum of the MFV axion potential is in zero and in consequence the QCD CP violating term exactly vanishes.

The MFV axion differs from the traditional QCD axion and from the so-called invisible axions [77-80] as the transformation properties under the axial $\mathrm{U}(1)$ factor are determined by the flavour structure of the SM fermions. Its associated phenomenology will be discussed in astrophysics, collider searches and in flavour observables, mainly focussing on meson decays.

The MFV axion manifests different signatures even with respect to the so-called Axiflavon or Flaxion, recently presented in refs. [81, 82], based on the pioneering paper in ref. [83]. The Axiflavon is the GB arising from the spontaneous breaking of the flavour $\mathrm{U}(1)_{\mathrm{FN}}$ symmetry in the FN mechanism and its distinctive feature resides in its flavour violating couplings. On the contrary, the MFV axion presents flavour conserving couplings, but violating the flavour universality. The predictions for meson decays are therefore different.

The rest of the letter is structured as follows. Few selected features of the MFV context are reported in section 2. The MFV axion is presented in section 3. Its phenomenology is discussed in section 4 together with a comparison with the Axiflavon. Conclusive remarks can be found in section 5. More technical details are reported in the appendix A.

\section{The minimal flavour violation revisited}

According to the modern realisation of MFV [41-45], the SM fermionic kinetic terms exhibit a $\mathrm{U}(3)^{5}$ flavour symmetry that can be decomposed into the product of an Abelian and a non-Abelian factor, $\mathcal{G}_{F}^{\mathrm{NA}} \times \mathcal{G}_{F}^{\mathrm{A}}$ where

$$
\begin{aligned}
\mathcal{G}_{F}^{\mathrm{NA}} & \equiv \mathrm{SU}(3)_{q_{L}} \times \mathrm{SU}(3)_{u_{R}} \times \mathrm{SU}(3)_{d_{R}} \times \mathrm{SU}(3)_{\ell_{L}} \times \mathrm{SU}(3)_{e_{R}} \\
\mathcal{G}_{F}^{\mathrm{A}} & \equiv \mathrm{U}(1)_{B} \times \mathrm{U}(1)_{L} \times \mathrm{U}(1)_{Y} \times \mathrm{U}(1)_{\mathrm{PQ}} \times \mathrm{U}(1)_{e_{R}} .
\end{aligned}
$$

In the previous expressions, $q_{L}$ and $\ell_{L}$ stand for the quark and lepton $\mathrm{SU}(2)_{L}$ doublets, while $u_{R}, d_{R}$ and $e_{R}$ for the quark and lepton singlets, each of them transforming as a triplet of the corresponding symmetry group; $B$ and $L$ refer to the Baryon and Lepton numbers, $Y$ to the Hypercharge, PQ to the PQ symmetry, while the last Abelian symmetry factor corresponds to rotations on only the $e_{R}$ fields.

In order to guarantee the invariance under this flavour symmetry of the entire SM Lagrangian, the Yukawa matrices $Y_{i}$ are promoted to spurion fields $\mathcal{Y}_{i}$ transforming under $\mathcal{G}_{F}^{\mathrm{NA}}$ as

$$
\mathcal{Y}_{u} \sim(\mathbf{3}, \overline{\mathbf{3}}, 1,1,1) \quad \mathcal{Y}_{d} \sim(\mathbf{3}, 1, \overline{\mathbf{3}}, 1,1) \quad \mathcal{Y}_{e} \sim(1,1,1, \mathbf{3}, \overline{\mathbf{3}})
$$


The Yukawa spurions then acquire background values, which explicitly break $\mathcal{G}_{F}^{\mathrm{NA}}$ and describe fermion masses and mixings. A free choice for these values is the ensamble of fermion masses and mixing angles, that can be written as follows:

$$
\begin{aligned}
& \left\langle\mathcal{Y}_{u}\right\rangle=c_{t} V^{\dagger} \operatorname{diag}\left(\frac{m_{u}}{m_{t}}, \frac{m_{c}}{m_{t}}, 1\right) \\
& \left\langle\mathcal{Y}_{d}\right\rangle=c_{b} \operatorname{diag}\left(\frac{m_{d}}{m_{b}}, \frac{m_{s}}{m_{b}}, 1\right) \\
& \left\langle\mathcal{Y}_{e}\right\rangle=c_{\tau} \operatorname{diag}\left(\frac{m_{e}}{m_{\tau}}, \frac{m_{\mu}}{m_{\tau}}, 1\right) .
\end{aligned}
$$

where $m_{i}$ are the fermion masses, $V$ is the CKM mixing matrix and $c_{i}$ are global numerical factors, not larger than $1 .^{2}$ Neutrino masses and the PMNS mixing matrix, although their introduction through the Seesaw mechanism is straightforward, will not be tackled in this letter as the focus is the MFV axion and the solution of the strong CP problem.

Refs. [64-67] showed how the non-Abelian symmetry $\mathcal{G}_{F}^{N A}$ deals exclusively with the explanation of the inter-generation hierarchies, but cannot fix the overall coefficients $c_{i}$. On the other side, the hierarchies $m_{b} / m_{t}$ and $m_{\tau} / m_{t}$, that correspond to $c_{b} / c_{t}$ and $c_{\tau} / c_{t}$ in the previous expressions, can be elegantly explain à la FN mechanism: one of the Abelian factors of the whole flavour symmetry can be taken as a true symmetry of the Lagrangian; charges can be chosen such that the only terms invariant under this Abelian factor are the ones of the up-type quarks, while those describing down-type quarks and charged leptons are initially forbidden. The latter terms originate only at the non-renormalisable level, after the addition in the scalar spectrum of a new field, the flavon $\Phi$, transforming under this Abelian factor, which re-establishes the invariance under the symmetry.

The minimality criterium in terms of field content identifies $\mathrm{U}(1)_{\mathrm{PQ}}$ as the only candidate, ${ }^{3}$ among the Abelian factors in $\mathcal{G}_{F}^{\mathrm{A}}$, to explain the intra-generation hierarchies: Baryon and Lepton numbers and Hypercharge are fixed by definition; $\mathrm{U}(1)_{e_{R}}$ does not affects down-type quarks and therefore would only explain the ratio $m_{\tau} / m_{t}$; although a double FN mechanism could be possible, taking both $\mathrm{U}(1)_{\mathrm{PQ}}$ and $\mathrm{U}(1)_{e_{R}}$ as true symmetries of the Lagrangian would lead to the introduction of two flavons, increasing the complexity of the model. Instead, as all the fermions potentially transform under $\mathrm{U}(1)_{\mathrm{PQ}}$, this choice allows to explain both the rations $m_{b} / m_{t}$ and $m_{\tau} / m_{t}$ by introducing a single scalar field. This is the strategy adopted in the following.

Without specifying, for sake of generality, the fermionic $\mathrm{U}(1)_{\mathrm{PQ}}$ charge assignment, the Yukawa Lagrangian reads as

$$
\mathscr{L}_{Y}=-\left(\frac{\Phi}{\Lambda_{\Phi}}\right)^{x_{u}-x_{q}} \bar{q}_{L} \widetilde{H} \mathcal{Y}_{u} u_{R}-\left(\frac{\Phi}{\Lambda_{\Phi}}\right)^{x_{d}-x_{q}} \bar{q}_{L} H \mathcal{Y}_{d} d_{R}-\left(\frac{\Phi}{\Lambda_{\Phi}}\right)^{x_{e}-x_{\ell}} \bar{\ell}_{L} H \mathcal{Y}_{e} e_{R}
$$

\footnotetext{
${ }^{2}$ Considering values of $c_{i}$ larger than 1 implies that multiple products of Yukawa spurions would be more relevant than the single spurions themselves, and then they should be treated in a non-perturbative approach as discussed in ref. [49].

${ }^{3}$ Ref. [84] discussed a similar context, where the non-Abelian terms of the flavour symmetry are gauged and two distinct Abelian factors are considered to explain the ratio between the third family quark masses. The spectrum and flavour gauge symmetries lead to a different phenomenology with respect to the one discussed in refs. [51, 55] and here.
} 
where $H$ is the Higgs $\mathrm{SU}(2)_{L}$ doublet, $\widetilde{H}=i \sigma_{2} H^{*}$, and $x_{i}$ are the PQ charges of the $i$ field. ${ }^{4}$ In this expression, the charge of the flavon $\Phi$ has been fixed to -1 , without loss of generality.

Once this flavon develops a VEV,$\langle\Phi\rangle \equiv v_{\Phi}$, and the Yukawa spurions acquire their background values, the Yukawa interactions read

$$
Y_{u}=\epsilon^{x_{u}-x_{q}}\left\langle\mathcal{Y}_{u}\right\rangle \quad Y_{d}=\epsilon^{x_{d}-x_{q}}\left\langle\mathcal{Y}_{d}\right\rangle \quad Y_{e}=\epsilon^{x_{e}-x_{\ell}}\left\langle\mathcal{Y}_{u}\right\rangle,
$$

where $\epsilon \equiv v_{\Phi} / \sqrt{2} \Lambda_{\Phi}$. It follows that the ratios between the third generation fermions are governed by the specific fermion PQ charge assignment:

$$
m_{b} / m_{t} \simeq \epsilon^{x_{d}-x_{u}} \quad m_{\tau} / m_{t} \simeq \epsilon^{x_{e}-x_{\ell}-x_{u}+x_{q}},
$$

where the ratios of the $c_{i}$ factors have been omitted as they are expected to be of the same order. The fact that the top mass is given by $c_{t} v / \sqrt{2}$ implies that $c_{t} \simeq 1$ and it results in selecting as the simplest choice

$$
x_{q}=x_{u}=0
$$

for the quark doublets and up-quark singlets PQ charges. In consequence, the other charges must undergo the following relations,

$$
x_{d} \simeq \log _{\epsilon}\left(m_{b} / m_{t}\right) \quad x_{e}-x_{\ell} \simeq \log _{\epsilon}\left(m_{\tau} / m_{t}\right)
$$

where $\epsilon$ is still an unknown quantity at this level. An exact value for this parameter depends on the specific ultraviolet theory that originates the low-energy Lagrangian in eq. (2.4). An estimation of the range of value it may acquire takes into consideration that $\epsilon$ should remain in the perturbative regime and that the value of $v_{\Phi}$ is expected to be not so much smaller than $\Lambda_{\Phi}$ (without a dynamical mechanism to explain it). In this letter, $\epsilon$ is taken in the interval [0.01, 0.3], consistently with previous studies on FN models $[6,7]$. The logarithm in eq. (2.8) softens the dependence on the exact value of $\epsilon$ : for $\epsilon$ inside its preferred interval, $x_{d}$ and $x_{e}-x_{\ell}$ are found in the range [1, 4]; to fix a reference value that will be used in the phenomenological analysis,

$$
x_{d}=3, \quad x_{e}-x_{\ell}=3,
$$

corresponding to $\epsilon \sim 0.23$, i.e. the Cabibbo angle.

Charged fermion masses and quark mixings do not help further to break the flat direction $x_{e}-x_{\ell}$. On the other side, neutrinos masses introduce an additional condition: describing neutrino mass terms via the Weinberg operator [85], invariance under $\mathrm{U}(1)_{\mathrm{PQ}}$ implies that this operator is written with $2 x_{\ell}$ insertions of the flavon $\Phi$,

$$
\mathscr{L}_{5}=\left(\frac{\Phi}{\Lambda_{\Phi}}\right)^{2 x_{\ell}} \times \frac{\left(\overline{\ell_{L}^{c}} \widetilde{H}^{*}\right) \mathscr{J}_{\nu}\left(\widetilde{H}^{\dagger} \ell_{L}\right)}{\Lambda_{L}},
$$

\footnotetext{
${ }^{4}$ The mixed use of a PQ flavon $\Phi$ and of the Yukawa spurions $\mathcal{Y}_{i}$ may be puzzling. Indeed, at this level of the discussion, it is equivalent introducing a dynamical flavon $\Phi$ or treating its effects via a PQ spurion (see ref. [44] for the latter case). Similarly, the Yukawa spurions may be promoted to be dynamical fields (see refs. [64-67]). The discussion that follows and the results presented in this section are not affected by this choice. Instead, the necessity to describe the breaking of the PQ symmetry through a dynamical flavon resides in the origin of the MFV axion, as it will be explained in the section 3.
} 
where $\Lambda_{L}$ is the scale of lepton number violation and $\mathscr{g}_{\nu}$ is the spurion field, transforming as $(1,1,1, \overline{6}, 1)$ under $\mathcal{G}_{F}^{\mathrm{NA}}$, whose background value $\left\langle\mathscr{g}_{\nu}\right\rangle \equiv g_{\nu}$ contains the information of the neutrino mass eigenvalues and the PMNS mixing matrix (see refs. [42, 45] for details). Larger values of $x_{\ell}$ implies that a lower scale $\Lambda_{L}$ is sufficient to explain the lightness of the active neutrinos. Moreover, requiring that the eigenvalues of $g_{\nu}$ are not larger than 1 (as for $c_{i}$ in eq. (2.3)), an upper bound on $\Lambda_{L}$ can be identified:

$$
\Lambda_{L} \simeq \frac{v^{2}}{2} \frac{g_{\nu} \epsilon^{2 x_{\ell}}}{\sqrt{\Delta m_{\mathrm{atm}}^{2}}} \lesssim 6 \times 10^{14} \mathrm{GeV} \times \epsilon^{2 x_{\ell}},
$$

where $\Delta m_{\mathrm{atm}}^{2}$ is the atmospheric neutrino mass squared difference. Ref. [45] (see figure 1) shows that, for $x_{\ell}=0$, the present data on the $\mu \rightarrow e$ conversion in golden nuclei largely excludes the parameter space for this model. A straightforward computation for $x_{\ell}=2$ and $\epsilon=0.23$ easily reveals that the parameter space is practically excluded. This suggests that only two values, $x_{\ell}=0$ and $x_{\ell}=1$, should be considered in the phenomenological analysis that follows. Summarising, two scenarios will be studied: ${ }^{5}$

$$
\begin{array}{lll}
\mathrm{S} 0: & x_{q}=0=x_{u}=x_{\ell}, & x_{d}=3=x_{e} \\
\mathrm{~S} 1: & x_{q}=0=x_{u}, \quad x_{\ell}=1, & x_{d}=3, \quad x_{e}=4 .
\end{array}
$$

\section{The MFV axion}

The origin of an axion in this context is associated to the PQ flavon: if $\Phi$ is a complex scalar field, then it contains two degrees of freedom, which in the polar coordinates can be expressed as follows: in the PQ broken phase,

$$
\Phi=\frac{\rho+v_{\Phi}}{\sqrt{2}} e^{i a / f_{a}}
$$

where $\rho$ is the radial component and $a$, which can be identified with the axion, is the the angular one.

The full scalar potential of the model presents three distinct parts:

$$
\mathscr{V}=-\mu^{2}|H|^{2}+\lambda|H|^{4}-\mu_{\Phi}^{2}|\Phi|^{2}+\lambda_{\Phi}|\Phi|^{4}+\lambda_{H \Phi}|H|^{2}|\Phi|^{2} .
$$

In a part of the parameter space, the pure $\Phi$-dependent scalar potential has a minimum corresponding to a non-vanishing VEV for $\Phi, v_{\Phi}^{2}=\mu_{\Phi}^{2} / \lambda_{\Phi}$. In the phenomenological section, it will be shown that $v_{\Phi}$ is necessarily large and this may represent a problem for the EW symmetry breaking (EWSB) mechanism: indeed the quartic $|H|^{2}|\Phi|^{2}$ coupling would contribute to the quadratic term of the pure $H$-dependent potential,

$$
\mu^{2} \rightarrow \mu^{\prime 2} \equiv \mu^{2}-\lambda_{H \Phi} v_{\Phi}^{2} .
$$

\footnotetext{
${ }^{5}$ The stability of a generic choice for these charges under the renormalisation group evolution has been discussed in ref. [86], specially considering the impact on axion couplings, which will be the subject of the next section. These effects could be relevant if the axion scale $f_{a}$ is relatively small, while for the values considered here they can be neglected.
} 
If no ad hoc cancellation between these two terms is invoked and for arbitrary values of $\lambda_{H \Phi}$, the new mass parameter $\mu^{\prime}$ resides at the same, large scale of $v_{\Phi}$. In order to reproduce the expected value of the EW VEV, $v \equiv 245 \mathrm{GeV}$ fixed through the $W$ gauge boson mass, it is then necessary to invoke a large value of the Higgs quartic coupling $\lambda$, describing in this way a strongly interacting scenario with a non-linearly realised EWSB mechanism. This is an intriguing possibility, especially considering the recent interest in non-SM descriptions of the Higgs sector, such as composite Higgs models [87-95], dilaton models [96-103], or general effective Lagrangians [104-121]. In this letter, however, the traditional EWSB mechanism will be considered, and this requires to invoke a fine tuning: either there is cancellation between $\mu^{2}$ and $\lambda_{H \Phi} v_{\Phi}^{2}$, or $\lambda_{H \Phi}$ is artificially small.

On the other side, a large $v_{\Phi}$ induces a large mass for the radial component $\rho$, that can be safely integrated out from the low-energy Lagrangian, leaving the axion $a$ as the unique light degree of freedom of $\Phi$.

The low-energy Lagrangian of the model can therefore be written as the sum of distinct terms:

$$
\begin{aligned}
\mathscr{L}= & \mathscr{L}_{K i n}^{\mathrm{SM}}+\frac{1}{2} \partial_{\mu} a \partial^{\mu} a+\mu^{\prime 2}|H|^{2}-\lambda|H|^{4}+ \\
& -e^{i\left(x_{u}-x_{q}\right) a / f_{a}} \bar{q}_{L} \widetilde{H} Y_{u} u_{R}-e^{i\left(x_{d}-x_{q}\right) a / f_{a}} \bar{q}_{L} H Y_{d} d_{R}+ \\
& -e^{i\left(x_{e}-x_{\ell}\right) a / f_{a}} \bar{\ell}_{L} H Y_{e} e_{R}+\frac{\alpha_{s}}{8 \pi} \theta_{\mathrm{QCD}} G^{a \mu \nu} \widetilde{G}_{\mu \nu}^{a} .
\end{aligned}
$$

Some comments are in order. The Yukawa matrices are the ones defined in eq. (2.5) and the specific choice of the PQ charges in eq. (2.12) has not been implemented yet, to keep general the discussion. The axion kinetic term is canonically normalised only after identifying its characteristic scale and the VEV of $\Phi$,

$$
f_{a} \equiv v_{\Phi}
$$

keeping $f_{a}$ in the Lagrangian to match the notation in the literature.

It is straightforward to perform axial transformations to the fermion fields in order to remove the axion dependence from the Yukawa terms. The resulting Lagrangian consists of the SM Lagrangian modified by the addition of interactions with the axion: while the complete list of terms can be found in appendix A, the axion couplings with fermions and gauge bosons field strengths in the physical basis read

$$
\begin{aligned}
\delta \mathscr{L} \supset & -c_{a \psi} \frac{\partial_{\mu} a}{2 f_{a}} \bar{\psi} \gamma^{\mu} \gamma_{5} \psi+\frac{\alpha_{s}}{8 \pi} \frac{a}{f_{a}} c_{a g g} G^{a \mu \nu} \widetilde{G}_{\mu \nu}^{a}+ \\
& +\frac{\alpha_{e m}}{8 \pi} \frac{a}{f_{a}} c_{a \gamma \gamma} F^{\mu \nu} \widetilde{F}_{\mu \nu}+\frac{\alpha_{e m}}{8 \pi} \frac{a}{f_{a}} c_{a Z Z} Z^{\mu \nu} \widetilde{Z}_{\mu \nu}+ \\
& +\frac{\alpha_{e m}}{8 \pi} \frac{a}{f_{a}} c_{a \gamma Z} F^{\mu \nu} \widetilde{Z}_{\mu \nu}+\frac{\alpha_{e m}}{8 \pi} \frac{a}{f_{a}} c_{a W W} W^{+\mu \nu} \widetilde{W}_{\mu \nu}^{-},
\end{aligned}
$$




\begin{tabular}{|c||c|c||c|c|c|c|c|c|c|c|}
\hline & $x_{\ell}$ & $x_{e}$ & $c_{a u}$ & $c_{a d}$ & $c_{a e}$ & $c_{a g g}$ & $c_{a \gamma \gamma}$ & $c_{a Z Z}$ & $c_{a \gamma Z}$ & $c_{a W W}$ \\
\hline$S 0$ & 0 & 3 & 0 & -3 & -3 & -9 & -24 & -6.8 & -12.8 & 0 \\
$S 1$ & 1 & 4 & 0 & -3 & -3 & -9 & -24 & +2.8 & -20 & +27 \\
\hline
\end{tabular}

Table 1. Values of the coefficients of the axion couplings to fermions and gauge boson field strengths in the physical basis for the two scenarios identified in eq. (2.12), where the normalisation is defined in eq. (3.6).

where $\psi=\{u, d, \nu, e\}$ and the explicit coefficients are

$$
\begin{aligned}
c_{a u} & =x_{q}-x_{u} \\
c_{a d} & =x_{q}-x_{d} \\
c_{a \nu} & =x_{\ell} \\
c_{a e} & =x_{\ell}-x_{e} \\
c_{a g g} & =3\left(2 x_{q}-x_{u}-x_{d}\right) \\
c_{a \gamma \gamma} & =2\left(5 x_{q}+3 x_{\ell}-4 x_{u}-x_{d}-3 x_{e}\right) \\
c_{a Z Z} & =t_{\theta}^{2}\left(x_{q}+3 x_{\ell}-8 x_{u}-2 x_{d}-6 x_{e}\right)+\frac{3}{t_{\theta}^{2}}\left(3 x_{q}+x_{\ell}\right) \\
c_{a \gamma Z} & =t_{\theta}\left(x_{q}+3 x_{\ell}-8 x_{u}-2 x_{d}-6 x_{e}\right)-\frac{3}{t_{\theta}}\left(3 x_{q}+x_{\ell}\right) \\
c_{a W W} & =\frac{6}{s_{\theta}^{2}}\left(x_{q}+x_{\ell}\right)
\end{aligned}
$$

with for shortness $t_{\theta} \equiv \tan \theta_{W}$ and $s_{\theta} \equiv \sin \theta_{W}$, being $\theta_{W}$ the Weinberg angle.

As anticipated in the introduction, the MFV axion solves the Strong CP problem in exactly the same way as the traditional QCD axion, i.e. absorbing the $\theta_{\mathrm{QCD}}$ parameter by a shift symmetry transformation. Only two conditions must be satisfied: the first is that $c_{\text {agg }} \neq 0$, which is consistent with eq. (2.12), that explains the top Yukawa coupling of order 1 and the smallness of the bottom to top mass ratio; the second is that the VEV of the redefined axion field is vanishing.

Table 1 reports the values of the $c_{a i}$ coefficients of the axion couplings to fermions and gauge field strengths in the physical basis for the two scenarios described in eq. (2.12). Of particular interest is that the ratio between the axion coupling to photons and that to gluons, which is typically a free parameter [122-127], is exactly fixed to $8 / 3$, as in the original DFSZ invisible axion model.

Notice that the common notation adopted in the literature makes use of effective couplings that can be expressed in terms of the $c_{a i}$ coefficients as follows:

$$
g_{a g g} \equiv \frac{\alpha_{s}}{2 \pi} \frac{c_{a g g}}{f_{a}} \quad g_{a i} \equiv \frac{\alpha_{\mathrm{em}}}{2 \pi} \frac{c_{a i}}{f_{a}},
$$

where $i=\{\gamma \gamma, Z Z, \gamma Z, W W\}$. 


\section{Phenomenological features}

Several studies have been performed to constrain the axion couplings to SM fermions and gauge bosons [128-153]. Two recent summaries can be found in refs. [154, 155]. These bounds strongly depend on the axion mass, that also determines the stability of the axion at collider sizes. The main results will be reported in this section, translating the distinct constraints into limits on the axion scale $f_{a}$.

Coupling to photons. Astrophysical, cosmological and low-energy terrestrial data provides the strongest bounds on axion couplings, those to photons (the latest constraints have been recently published in ref. [152]): the upper bounds on the effective couplings can be summed up as $[154,155]$

$$
\begin{aligned}
& \left|g_{a \gamma \gamma}\right| \lesssim 7 \times 10^{-11} \mathrm{GeV}^{-1} \text { for } m_{a} \lesssim 10 \mathrm{meV} \\
& \left|g_{a \gamma \gamma}\right| \lesssim 10^{-10} \mathrm{GeV}^{-1} \quad \text { for } 10 \mathrm{meV} \lesssim m_{a} \lesssim 10 \mathrm{eV} \\
& \left|g_{a \gamma \gamma}\right| \ll 10^{-12} \mathrm{GeV}^{-1} \quad \text { for } 10 \mathrm{eV} \lesssim m_{a} \lesssim 0.1 \mathrm{GeV} \\
& \left|g_{a \gamma \gamma}\right| \lesssim 10^{-3} \mathrm{GeV}^{-1} \quad \text { for } 0.1 \mathrm{GeV} \lesssim m_{a} \lesssim 1 \mathrm{TeV} \text {. }
\end{aligned}
$$

Notice that the bounds for masses between $10 \mathrm{eV}$ and $0.1 \mathrm{GeV}$, which include the so-called $\mathrm{MeV}$ window, come from (model dependent) cosmological data [144]. On the other side, for masses larger than the $\mathrm{TeV}$, no constraint is present. These bounds can be translated in terms of $f_{a}$ through eq. (3.8): taking $\alpha_{\mathrm{em}}=1 / 137.036$,

$$
\begin{array}{ll}
f_{a} \gtrsim 4 \times 10^{8} \mathrm{GeV} & \text { for } m_{a} \lesssim 10 \mathrm{meV} \\
f_{a} \gtrsim 2.8 \times 10^{8} \mathrm{GeV} & \text { for } 10 \mathrm{meV} \lesssim m_{a} \lesssim 10 \mathrm{eV} \\
f_{a} \gg 2.8 \times 10^{10} \mathrm{GeV} & \text { for } 10 \mathrm{eV} \lesssim m_{a} \lesssim 0.1 \mathrm{GeV} \\
f_{a} \gtrsim 28 \mathrm{GeV} & \text { for } 0.1 \mathrm{GeV} \lesssim m_{a} \lesssim 1 \mathrm{TeV} .
\end{array}
$$

These results hold for both the $S 0$ and $S 1$ scenarios, as $c_{a \gamma \gamma}$ does not depend on the specific value chosen for $x_{\ell}$.

Coupling to gluons. Collider mono-jet searches [141, 142, 146, 150] and axion-pion mixing effects $[128,130]$ allows to extract bounds on the axion couplings with gluons:

$$
\begin{array}{ll}
\left|g_{\text {agg }}\right| \lesssim 1.1 \times 10^{-5} \mathrm{GeV}^{-1} & \text { for } m_{a} \lesssim 60 \mathrm{MeV} \\
\left|g_{\text {agg }}\right| \lesssim 10^{-4} \mathrm{GeV}^{-1} & \text { for } 60 \mathrm{MeV} \lesssim m_{a} \lesssim 0.1 \mathrm{GeV}
\end{array}
$$

that can be translated into constraints on $f_{a}$,

$$
\begin{array}{ll}
f_{a} \gtrsim 15.4 \times 10^{3} \mathrm{GeV} & \text { for } m_{a} \lesssim 60 \mathrm{MeV} \\
f_{a} \gtrsim 1.7 \times 10^{3} \mathrm{GeV} & \text { for } 60 \mathrm{MeV} \lesssim m_{a} \lesssim 0.1 \mathrm{GeV}
\end{array}
$$

taking $\alpha_{s}\left(M_{Z}^{2}\right)=0.1184$. Also for $c_{a g g}$, there is no dependence on the specific value of $x_{\ell}$. 
Couplings to massive gauge bosons (collider). Considering LHC data with $\sqrt{s}=$ $13 \mathrm{TeV}$, dedicated analyses on Mono-W $\left(p p \rightarrow a W\left(W \rightarrow \mu \nu_{\mu}\right)\right)$ mono-Z $(p p \rightarrow a Z(Z \rightarrow$ ee)) channels and the $Z$ boson width put bounds on axion couplings to two $W$ 's, to two $Z$ 's and to $\gamma Z$ : for $m_{a} \lesssim 1 \mathrm{GeV}[150]$,

$$
\begin{aligned}
\left|g_{a W W}\right| & \lesssim 5 \times 10^{-7} \mathrm{GeV}^{-1} \\
\left|g_{a Z Z}\right| & \lesssim 3 \times 10^{-7} \mathrm{GeV}^{-1} \\
\left|g_{a Z \gamma}\right| & \lesssim 1.8 \times 10^{-3} \mathrm{GeV}^{-1} .
\end{aligned}
$$

In the $S 0$ scenario, $c_{a W W}$ is identically vanishing and therefore the corresponding bound is automatically satisfied. Instead, the bounds on the $a Z Z$ and $a Z \gamma$ effective couplings translate into the following constraints on $f_{a}$ :

$$
\begin{array}{ll}
(a Z Z) & f_{a} \gtrsim 2.6 \times 10^{4} \mathrm{GeV} \\
(a Z \gamma) & f_{a} \gtrsim 8.3 \mathrm{GeV} .
\end{array}
$$

Different results holds for the $S 1$ scenario, where $x_{\ell} \neq 0$ :

$$
\begin{array}{ll}
(a W W) & f_{a} \gtrsim 6.3 \times 10^{4} \mathrm{GeV} \\
(a Z Z) & f_{a} \gtrsim 1.1 \times 10^{4} \mathrm{GeV} \\
(a Z \gamma) & f_{a} \gtrsim 12.9 \mathrm{GeV} .
\end{array}
$$

Couplings to fermions and $\boldsymbol{W}$ 's (flavour). Studies on Compton scattering of axions in the Sun, axionic recombination and de-excitation in iones and axion bremsstrahlung [136] set very strong bounds on axion couplings to electrons for masses below $\sim 80 \mathrm{keV}$. Similar constraints are inferred from Compton conversion of solar axions [133] for masses up to $\sim 10 \mathrm{MeV}$. All together, the axion coupling to electrons is bounded to be

$$
\frac{c_{a e}}{f_{a}} \lesssim 5.2 \times 10^{-8} \mathrm{GeV}^{-1} \text { for } 1 \mathrm{eV} \lesssim m_{a} \lesssim 10 \mathrm{MeV}
$$

Even more stringent bounds arise from observation of Red Giants [138], but for a smaller range of masses:

$$
\frac{c_{a e}}{f_{a}} \lesssim 8.6 \times 10^{-10} \mathrm{GeV}^{-1} \text { for } m_{a} \lesssim 1 \mathrm{eV} .
$$

When considering the explicit value of the $c_{a e}$ coefficient, that is the same for the two PQ charge scenarios, these constraints translate into bounds on the axion scale:

$$
\begin{aligned}
& f_{a} \gtrsim 3.5 \times 10^{9} \mathrm{GeV} \text { for } m_{a} \lesssim 1 \mathrm{eV} \\
& f_{a} \gtrsim 5.8 \times 10^{7} \mathrm{GeV} \text { for } 1 \mathrm{eV} \lesssim m_{a} \lesssim 10 \mathrm{MeV}
\end{aligned}
$$

Rare meson decays provide strong constraints of axion couplings to quarks and to two $W$ gauge bosons. For masses below $\sim 0.2 \mathrm{GeV}$, the most relevant observable is $K^{+} \rightarrow$ $\pi^{+}+a$, whose branching ratio undergoes to the following limit [132]:

$$
B R_{K^{+} \rightarrow \pi^{+}+a}<7.3 \times 10^{-11} .
$$


For larger masses up to a few $\mathrm{GeVs}$, the $B^{+} \rightarrow K^{+}+a$ decay provides the most stringent bound [135]:

$$
B R_{B^{+} \rightarrow K^{+}+a}<3.2 \times 10^{-5} .
$$

In other analyses $[149,150,155]$, meson-to-axion decays, where the latter subsequently decays into two photons, are also considered: these observables are however inconclusive for the MFV axion model because the constraints on $a \gamma \gamma$ coupling are so strong that prevent any present or future signal at experiments for this class of observables.

The two PQ charge assignment scenarios lead to distinct results when considering these observables. As the axion does not couple to up-type quarks $\left(c_{a u}=0\right)$, the two decays $K^{+} \rightarrow \pi^{+}+a$ and $B^{+} \rightarrow K^{+}+a$ can only occur at 1-loop level with the axion arising from the interaction with the internal $W$ propagator, as for the $S 1$ scenario. The constraints that can be deduced on $g_{a W W}$ read as [149]:

$$
\begin{array}{ll}
\left|g_{a W W}\right| \lesssim 3 \times 10^{-6} \mathrm{GeV}^{-1} & \text { for } m_{a} \lesssim 0.2 \mathrm{GeV} \\
\left|g_{a W W}\right| \lesssim 10^{-4} \mathrm{GeV}^{-1} & \text { for } 0.2 \mathrm{GeV} \lesssim m_{a}
\end{array}
$$

that can be translated in terms of $f_{a}$ expliciting the value of $c_{a W W}$,

$$
\begin{aligned}
& f_{a} \gtrsim 10^{5} \mathrm{GeV} \\
& f_{a} \gtrsim 3.1 \times 10^{2} \mathrm{GeV}
\end{aligned}
$$

$$
\begin{aligned}
& \text { for } m_{a} \lesssim 0.2 \mathrm{GeV} \\
& \text { for } 0.2 \mathrm{GeV} \lesssim m_{a} \lesssim 5 \mathrm{GeV} .
\end{aligned}
$$

On the other side, in the $S 0$ scenario, $c_{a W W}$ is vanishing and then these two decays are not generated even at 1-loop (but they should arise at the 2-loop level).

The axion mass and the ALP scenario. Without an explicit soft breaking source of the shift symmetry, a mass term for the MFV axion may arise, as for the traditional QCD axion, from non-perturbative dynamics: the axion mixing with neutral mesons induces a contribution which is estimated to be $[78,156,157]$

$$
m_{a} \sim 6 \mu \mathrm{eV}\left(\frac{10^{12} \mathrm{GeV}}{f_{a}}\right)
$$

and not much larger than a few eV. Additional contributions may arise à la KSVZ axion in the presence of exotic fermions that couple to the axion. Exotic fermions are typically present when constructing the underlying theory originating the effective terms in eq. (2.4) (see for example ref. [18]) or are required from anomaly cancellation conditions in models with gauged flavour symmetries [51-55, 61, 62]: the largest mass contribution originated in these cases is of hundreds of $\mathrm{eV}$, for values of the axion scale $f_{a}$ close to the $\mathrm{TeV}$. Even considering possible contributions of this kind, one can safely conclude that the MFV axion mass is smaller than the keV, unless explicit shift symmetry breaking sources are introduced in the scalar potential. For these mass values the strongest constraints arise from the axion coupling to photons, eq. (4.2), and to electrons, eq. (4.10): the axion scale is necessarily larger than $\sim 10^{10} \mathrm{GeV}$ and $\sim 10^{9} \mathrm{GeV}$, preventing any possibility to detect the MFV axion at colliders or in flavour searches. This represents a new example of invisible axion that solves the Strong CP problem as in the traditional QCD axion model: the $\theta_{\mathrm{QCD}}$ 
is reabsorbed by a shift symmetry transformation and the Lagrangian term in eq. (1.1) is identically vanishing as the VEV of the redefined axion is zero. Perturbations to this solution may arise due to the running from the scale of PQ symmetry breaking down to the QCD scale and/or due to new physics contributions at high scale: however, the largeness of $f_{a}$ implies that these perturbations would be necessarily tiny. Other irreducible sources of explicit breaking are related to quantum gravity effects: they have been discussed for example in refs. [158-161] and are shown to be negligible under generic conditions.

On the other side, if a signal of detection that may be interpreted in terms of an axion is seen, it may be compatible with the MFV axion at the price of invoking an explicit breaking of the shift symmetry: in this case, the relation between the axion mass and its scale gets broken and the bounds aforementioned may be avoided. In the common language, this eventuality is refereed to as Axion-like-particle (ALP) framework, that received much attention from the community in the last years. This type of axions may not solve the Strong CP problem as the QCD scalar potential typically receives relevant contributions in this case: to solve the Strong CP problem it would be necessary that the scalar potential at high-energy allows a vanishing VEV at the minimum and that this configuration is maintained at the QCD scale. This mechanism is more general than the MFV axion under consideration and will be investigated in a separate project [162].

In what follows, this last scenario will be considered, assuming a MFV axion mass much larger than the eV region. For masses of the order of the $\mathrm{GeV}$, the strong bounds from the $a \gamma \gamma$ and aee couplings are easily evaded, and the next most sensitive observables are those from collider. For even larger masses, no bounds at all have been put.

Increasing the axion mass, however, its decay length decreases, and the axion may not be considered anymore stable at collider size and dedicated analyses should be in order. The distance travelled by an axion after being produced can be casted in the following expression [150]:

$$
d \approx \frac{10^{4}}{c_{a i}^{2}}\left(\frac{\mathrm{MeV}}{m_{a}}\right)^{4}\left(\frac{f_{a}}{\mathrm{GeV}}\right)^{2}\left(\frac{\left|p_{a}\right|}{\mathrm{GeV}}\right) \mathrm{m},
$$

where the typical momentum considered is of $100 \mathrm{GeV}$. Selecting a benchmark region with $m_{a} \simeq 1 \mathrm{GeV}$ and $f_{a} \gtrsim 60 \mathrm{TeV}$, as suggested by the collider bounds on $c_{a W W}$ in eq. (4.7), its decay length is larger than hundreds of meters and is practically stable at colliders.

For this benchmark region, all the bounds aforementioned are satisfied, including those from rare meson decays: in the $S 1$ scenario, where $c_{a W W} \neq 0$, the branching ratio for $B^{+} \rightarrow K^{+}+a$ decay (the $K^{+} \rightarrow \pi^{+}+a$ decay is kinematically forbidden) is predicted to be $\lesssim 10^{-13}$, much smaller than the expected future sensitivity of Belle II [163]. In the $S 0$ scenario, the vanishing of both $c_{a u}$ and $c_{a W W}$ implies that this process cannot occur at 1-loop, but only at 2-loop level, and therefore the predicted branching ratio is even smaller. Even turning the attention to processes that receive 1-loop contributions with down-type quark in the internal lines, such as $D$-meson hadronic decays, no interesting bound can be extracted: indeed, the $D^{+} \rightarrow \pi^{+}+a$ and $D_{s}^{+} \rightarrow K^{+}+a$ decays are proportional to $c_{a d}$ in the $S 0$ scenario, or to a combination of $c_{a d}$ and $c_{a W W}$ in the $S 1$ scenario, and therefore are predicted to be non-vanishing in both the considered scenarios. However, for the 
benchmark region identified above, the branching ratios of these processes are smaller than $10^{-15}$ and $10^{-16}$, which means that they are effects impossible to be seen experimentally.

Comparison with the Axiflavon. The Axiflavon [81, 82] is the axion arising in the context of the FN mechanism and has flavour violating couplings, in the mass basis for fermions, predicted in terms of the FN charges, up to $\mathcal{O}(1)$ uncertainties. This represents a major difference with respect to the MFV axion: the presence of flavour violating couplings induces tree-level flavour changing neutral current processes, such as the meson decays described in the previous section. In consequence, the Axiflavon is more sensitive to the scale of new physics than the MFV axion. To satisfy the present bounds on $K$ and $B$ decays, the axion scale $f_{a}$ needs to be of the order of $10^{10} \mathrm{GeV}$ [82], that approximatively coincides with the values necessary to pass the very stringent bounds on the $a \gamma \gamma$ and aee couplings. The Axiflavon is therefore an example of visible QCD axion, as it predicts lowenergy flavour effects, despite of the very large value of the axion scale $f_{a}$. On the other side, no signals are expected at colliders, as indeed effects in mono- $\mathrm{W}$ and mono- $\mathrm{Z}$ channels, and in the $Z$ boson width are expected to be tiny and not appreciable in the future phases of LHC. As a final comment that helps distinguishing between the MFV axion and the Axiflavon is the prediction for the ratio between the axion coupling to photons and that to gluons: in the first model this ratio is strictly predicted to be $8 / 3$, while in the second one it may vary within the range $[2.4,3]$.

\section{Conclusions}

The MFV ansatz, beside leading to very predictive context to solve the BSM flavour problem, is a fascinating approach to attempt to explain the flavour puzzle. Besides the nonAbelian parts of the full flavour symmetry, the Abelian terms may be responsible for explaining the mass hierarchies between the third fermion families. The fermion charge assignment is however not vectorial and this opens the possibility to interpret the Goldstone boson arising from the spontaneous breaking of one of these terms as an axion that solves the Strong CP problem.

The MFV axion couplings are determined by the fermion charge assignments, which are almost all fixed by requiring that $m_{t} \sim v / \sqrt{2}, m_{b} / m_{t}$ and $m_{\tau} / m_{t}$ fit the measured values, and the predicted value for $\mu \rightarrow e$ conversion in golden nuclei does not saturate the present experimental bound. The axion couplings with up-type quarks are identically vanishing, while those with down-type quarks, charged leptons, two gluons, two photons, two $Z$ 's, $Z \gamma$ are all non-vanishing and fixed to specific values. The only freedom left is in the value of the axion coupling to two $W$ 's: the choice for the charge of the lepton doublet $x_{\ell}$ is not unique, but it can take the value $x_{\ell}=0$, that identified the scenario $S 0$ where $a W W$ couplings is also vanishing, or the value $x_{\ell}=1$, dubbed as $S 1$ scenario where the MFV axion does couple to two $W$ 's.

The most constraining bounds affect the axion couplings to photons and to electrons: all in all, the axion scale $f_{a}$ needs to be larger than $10^{8} \mathrm{GeV}$ for masses smaller than $0.1 \mathrm{GeV}$. If follows that a MFV axion with masses below this value can be considered an 
invisible axion, as no effects are expected neither in low-energy experiments nor at colliders. With respect to other invisible axion models, such as DFSZ or KSVZ, the MFV axion has the advantages that its origin is not linked to an ad hoc introduction of the PQ symmetry, but follows naturally from an Abelian term of the SM flavour symmetry.

If a signal is seen in present or near future experiments that may be interpreted in terms of an axion, it would still be compatible with the MFV axion, but at the price of breaking the proportionality between its mass and its characteristic scale, invoking an explicit shift symmetry breaking. Indeed, for $m_{a} \simeq 1 \mathrm{GeV}$ and $f_{a} \gtrsim 60 \mathrm{TeV}$, the bounds from $a \gamma \gamma$ and aee couplings do not apply, opening the possibility of low-energy signals. The next most sensitive observables are those at colliders, and in particular mono- $\mathrm{W}$ and mono- $\mathrm{Z}$ channels and modifications of the prediction for the $Z$-decay width are very promising. On the contrary, flavour processes, the most constraining being $B \rightarrow K+a$ decay $(K \rightarrow \pi+a$ one is forbidden kinematically), are extremely suppressed and much below the sensitivities expected at Belle II. This is due to the fact that the MFV axion couplings are flavour conserving, but flavour universality violating: in consequence, this axion does not give rise to flavour changing neutral current processes at tree level, but describes flavour changing processes at 1-loop level. This represents the major difference with respect to the Axiflavon model, where the axion does violate flavour and describes rare meson decays at tree level: the existing bounds from $K \rightarrow \pi+a$ and $B \rightarrow K+a$ decays constrain the Axiflavon scale to be larger than $10^{10} \mathrm{GeV}$ : for these values the bounds on $a \gamma \gamma$ and aee are satisfied, while all the effects at colliders are expected to be tiny and far from the expected future improvements.

To summarise, if no signal at all will appear neither at colliders nor in low-energy flavour experiments, then the only possibility is the one of an invisible axion with a very large scale $f_{a}$, being the DFSZ, the KSVZ, the MFV axion and the Axiflavon all equally viable. The Strong CP problem can be solved by a shift symmetry transformation that absorbes the $\theta_{\mathrm{QCD}}$ parameter, with the QCD scalar potential that fixes to zero the VEV of the shifted axion.

If a signal emerges only at colliders, then this would be in favour of a heavy MFV axion, while disfavouring the Axiflavon. In this case, a precise measure of the axion couplings to photons and to gluons may be a smoking gun for the MFV axion model as the ratio between these two couplings is strictly predicted to be 8/3. A drawback of a heavy MFV axion is that the solution of the Strong CP problem is not guaranteed.

On the contrary, if a signal is seen only in rare flavour observables, then it indicates that the Axiflavon may be the correct axion candidate, while the MFV axion (light or heavy) would be disfavoured. Finally, if both type of signals are found, both at colliders and in rare meson decays, none of the two models would be able to naturally account for these events.

\section{Acknowledgments}

We thank Enrique Fernández Martínez and Emmanuel Stamou for enjoyable brainstorming during the development of this project and Paride Paradisi and Carlos Pena Ruano 
for valuable conversations on meson decays and flavoured axion. L.M. thanks the department of Physics and Astronomy of the Università degli Studi di Padova and the Fermilab Theory Division for hospitality during the writing up of this paper. L.M. acknowledges partial financial support by the European Union's Horizon 2020 research and innovation programme under the Marie Sklodowska-Curie grant agreements No 690575 and No 674896, by the Spanish MINECO through the "Ramón y Cajal" programme (RYC-2015-17173), and by the Spanish "Agencia Estatal de Investigación" (AEI) and the EU "Fondo Europeo de Desarrollo Regional" (FEDER) through the project FPA2016-78645-P, and through the Centro de excelencia Severo Ochoa Program under grant SEV-2016-0597.

\section{A MFV axion transformations}

Performing a fermion field redefinition in the Lagrangian in eq. (3.4),

$$
\begin{array}{rlrl}
q_{L} & \rightarrow q_{L} e^{-i x_{q} a / f_{a}} & \ell_{L} \rightarrow \ell_{L} e^{-i x_{\ell} a / f_{a}} \\
u_{R} \rightarrow u_{R} e^{-i x_{u} a / f_{a}} & e_{R} \rightarrow e_{R} e^{-i x_{e} a / f_{a}} \\
d_{R} \rightarrow d_{R} e^{-i x_{d} a / f_{a}} & &
\end{array}
$$

the axion couplings to the Yukawa terms are removed. The resulting Lagrangian is the sum of two terms, the SM one and a part containing all the axion couplings, $\delta \mathscr{L}$ :

$$
\mathscr{L} \rightarrow \mathscr{L}_{S M}+\delta \mathscr{L}
$$

where

$$
\begin{aligned}
\delta \mathscr{L}= & \frac{1}{2} \partial_{\mu} a \partial^{\mu} a-\sum_{\Psi} \frac{\partial_{\mu} a}{2 f_{a}} \bar{\Psi} \gamma^{\mu} \gamma_{5} \Psi\left(L_{\Psi}-R_{\Psi}\right)+ \\
& +\frac{3 g^{\prime 2}}{16 \pi^{2}} \frac{a}{f_{a}} B^{\mu \nu} \widetilde{B}_{\mu \nu}\left(\frac{1}{6} x_{Q}-\frac{4}{3} x_{u}-\frac{1}{3} x_{d}+\frac{1}{2} x_{L}-x_{e}\right)+ \\
& +\frac{3 g^{2}}{32 \pi^{2}} \frac{a}{f_{a}} W^{a \mu \nu} \widetilde{W}_{\mu \nu}^{a}\left(3 x_{Q}+x_{L}\right)+\frac{3 g_{s}^{2}}{32 \pi^{2}} \frac{a}{f_{a}} G^{a \mu \nu} \widetilde{G}_{\mu \nu}^{a}\left(2 x_{Q}-x_{u}-x_{d}\right) .
\end{aligned}
$$

It is useful to re-express this Lagrangian in the physical basis for the gauge bosons. Using the following notation for the gauge field strengths, $F_{\mu \nu}=\partial_{\mu} A_{\nu}-\partial_{\nu} A_{\mu}$ and $Z_{\mu \nu}=$ $\partial_{\mu} Z_{\nu}-\partial_{\nu} Z_{\mu}$, and the same goes for $W^{ \pm}$, the redefined Lagrangian reads as:

$$
\begin{aligned}
\delta \mathscr{L}= & -\sum_{\psi} c_{a \psi \psi} \frac{\partial_{\mu} a}{2 f_{a}} \bar{\psi} \gamma^{\mu} \gamma_{5} \psi+c_{a g g} \frac{\alpha_{s}}{8 \pi} \frac{a}{f_{a}} G^{a \mu \nu} \widetilde{G}_{\mu \nu}^{a}+ \\
& +\frac{\alpha_{e m}}{8 \pi} \frac{a}{f_{a}} c_{a \gamma \gamma} F^{\mu \nu} \widetilde{F}_{\mu \nu}+\frac{\alpha_{e m}}{8 \pi} \frac{a}{f_{a}} c_{a Z Z} Z^{\mu \nu} \widetilde{Z}_{\mu \nu}+ \\
& +\frac{\alpha_{e m}}{8 \pi} \frac{a}{f_{a}} c_{a \gamma Z} F^{\mu \nu} \widetilde{Z}_{\mu \nu}+\frac{\alpha_{e m}}{8 \pi} \frac{a}{f_{a}} c_{a W W} W^{+\mu \nu} \widetilde{W}_{\mu \nu}^{-}+ \\
& +\frac{\alpha_{e m}}{8 \pi} c_{a W W \gamma} a \partial^{\mu}\left(W^{-\nu} W^{+\rho}\right) A^{\sigma} \varepsilon_{\mu \nu \rho \sigma}+ \\
& +\frac{\alpha_{e m}}{8 \pi} c_{a W W Z} a \partial^{\mu}\left(W^{-\nu} W^{+\rho}\right) Z^{\sigma} \varepsilon_{\mu \nu \rho \sigma}+ \\
& -\frac{\alpha_{e m}}{8 \pi} c_{a W W W W} a W^{+\mu} W^{-\nu} W^{+\rho} W^{-\sigma} \varepsilon_{\mu \nu \rho \sigma},
\end{aligned}
$$


where all the coefficients $c_{a i}$ have been defined in eq. (3.7), with the exception of the last three that are defined as

$$
\begin{aligned}
c_{a W W \gamma} & =\frac{i 12 e}{s_{\theta}^{2} f_{a}}\left(3 x_{q}+x_{\ell}\right), \\
c_{a W W Z} & =\frac{i 12 c_{\theta} e}{s_{\theta}^{3} f_{a}}\left(3 x_{q}+x_{\ell}\right), \\
c_{a W W W W} & =\frac{6 e^{2}}{s_{\theta}^{4} f_{a}}\left(3 x_{q}+x_{\ell}\right),
\end{aligned}
$$

with $e$ the electron electric charge and $c_{\theta} \equiv \cos \theta_{W}$ for short.

Open Access. This article is distributed under the terms of the Creative Commons Attribution License (CC-BY 4.0), which permits any use, distribution and reproduction in any medium, provided the original author(s) and source are credited.

\section{References}

[1] C.D. Froggatt and H.B. Nielsen, Hierarchy of Quark Masses, Cabibbo Angles and CP-violation, Nucl. Phys. B 147 (1979) 277 [InSPIRE].

[2] G. Altarelli, F. Feruglio and I. Masina, From minimal to realistic supersymmetric SU(5) grand unification, JHEP 11 (2000) 040 [hep-ph/0007254] [INSPIRE].

[3] G. Altarelli, F. Feruglio and I. Masina, Models of neutrino masses: Anarchy versus hierarchy, JHEP 01 (2003) 035 [hep-ph/0210342] [INSPIRE].

[4] P.H. Chankowski, K. Kowalska, S. Lavignac and S. Pokorski, Update on fermion mass models with an anomalous horizontal U(1) symmetry, Phys. Rev. D 71 (2005) 055004 [hep-ph/0501071] [INSPIRE].

[5] W. Buchmüller, V. Domcke and K. Schmitz, Predicting $\theta_{13}$ and the Neutrino Mass Scale from Quark Lepton Mass Hierarchies, JHEP 03 (2012) 008 [arXiv:1111.3872] [INSPIRE].

[6] G. Altarelli, F. Feruglio, I. Masina and L. Merlo, Repressing Anarchy in Neutrino Mass Textures, JHEP 11 (2012) 139 [arXiv:1207.0587] [INSPIRE].

[7] J. Bergstrom, D. Meloni and L. Merlo, Bayesian comparison of U(1) lepton flavor models, Phys. Rev. D 89 (2014) 093021 [arXiv:1403.4528] [INSPIRE].

[8] E. Ma and G. Rajasekaran, Softly broken $A_{4}$ symmetry for nearly degenerate neutrino masses, Phys. Rev. D 64 (2001) 113012 [hep-ph/0106291] [INSPIRE].

[9] K.S. Babu, E. Ma and J.W.F. Valle, Underlying $A_{4}$ symmetry for the neutrino mass matrix and the quark mixing matrix, Phys. Lett. B 552 (2003) 207 [hep-ph/0206292] [INSPIRE].

[10] G. Altarelli and F. Feruglio, Tri-bimaximal neutrino mixing from discrete symmetry in extra dimensions, Nucl. Phys. B 720 (2005) 64 [hep-ph/0504165] [INSPIRE].

[11] G. Altarelli and F. Feruglio, Tri-bimaximal neutrino mixing, $A_{4}$ and the modular symmetry, Nucl. Phys. B 741 (2006) 215 [hep-ph/0512103] [INSPIRE].

[12] F. Feruglio, C. Hagedorn, Y. Lin and L. Merlo, Tri-bimaximal Neutrino Mixing and Quark Masses from a Discrete Flavour Symmetry, Nucl. Phys. B 775 (2007) 120 [Erratum ibid. B 836 (2010) 127] [hep-ph/0702194] [INSPIRE]. 
[13] F. Bazzocchi, L. Merlo and S. Morisi, Fermion Masses and Mixings in a S(4)-based Model, Nucl. Phys. B 816 (2009) 204 [arXiv:0901.2086] [INSPIRE].

[14] F. Bazzocchi, L. Merlo and S. Morisi, Phenomenological Consequences of See-Saw in $S_{4}$ Based Models, Phys. Rev. D 80 (2009) 053003 [arXiv:0902.2849] [InSPIRE].

[15] G. Altarelli, F. Feruglio and L. Merlo, Revisiting Bimaximal Neutrino Mixing in a Model with $S_{4}$ Discrete Symmetry, JHEP 05 (2009) 020 [arXiv:0903.1940] [INSPIRE].

[16] R. de Adelhart Toorop, F. Bazzocchi and L. Merlo, The Interplay Between GUT and Flavour Symmetries in a Pati-Salam $\times S_{4}$ Model, JHEP 08 (2010) 001 [arXiv:1003.4502] [INSPIRE].

[17] G. Altarelli and F. Feruglio, Discrete Flavor Symmetries and Models of Neutrino Mixing, Rev. Mod. Phys. 82 (2010) 2701 [arXiv: 1002.0211] [INSPIRE].

[18] I. de Medeiros Varzielas and L. Merlo, Ultraviolet Completion of Flavour Models, JHEP 02 (2011) 062 [arXiv:1011.6662] [InSPIRE].

[19] R. de Adelhart Toorop, F. Feruglio and C. Hagedorn, Discrete Flavour Symmetries in Light of T2K, Phys. Lett. B 703 (2011) 447 [arXiv:1107.3486] [inSPIRE].

[20] W. Grimus and P.O. Ludl, Finite flavour groups of fermions, J. Phys. A 45 (2012) 233001 [arXiv: 1110.6376] [INSPIRE].

[21] R. de Adelhart Toorop, F. Feruglio and C. Hagedorn, Finite Modular Groups and Lepton Mixing, Nucl. Phys. B 858 (2012) 437 [arXiv:1112.1340] [InSPIRE].

[22] S.F. King and C. Luhn, $A_{4}$ models of tri-bimaximal-reactor mixing, JHEP 03 (2012) 036 [arXiv:1112.1959] [INSPIRE].

[23] G. Altarelli, F. Feruglio and L. Merlo, Tri-Bimaximal Neutrino Mixing and Discrete Flavour Symmetries, Fortsch. Phys. 61 (2013) 507 [arXiv: 1205.5133] [InSPIRE].

[24] F. Bazzocchi and L. Merlo, Neutrino Mixings and the S4 Discrete Flavour Symmetry, Fortsch. Phys. 61 (2013) 571 [arXiv:1205.5135] [inSPIRE].

[25] S.F. King and C. Luhn, Neutrino Mass and Mixing with Discrete Symmetry, Rept. Prog. Phys. 76 (2013) 056201 [arXiv:1301.1340] [InSPIRE].

[26] F. Feruglio, C. Hagedorn, Y. Lin and L. Merlo, Lepton Flavour Violation in Models with $A_{4}$ Flavour Symmetry, Nucl. Phys. B 809 (2009) 218 [arXiv:0807.3160] [INSPIRE].

[27] F. Feruglio, C. Hagedorn and L. Merlo, Vacuum Alignment in SUSY $A_{4}$ Models, JHEP 03 (2010) 084 [arXiv:0910.4058] [INSPIRE].

[28] Y. Lin, L. Merlo and A. Paris, Running Effects on Lepton Mixing Angles in Flavour Models with Type I Seesaw, Nucl. Phys. B 835 (2010) 238 [arXiv:0911.3037] [InSPIRE].

[29] F. Feruglio, C. Hagedorn, Y. Lin and L. Merlo, Lepton Flavour Violation in a Supersymmetric Model with $A_{4}$ Flavour Symmetry, Nucl. Phys. B 832 (2010) 251 [arXiv:0911.3874] [INSPIRE].

[30] H. Ishimori, T. Kobayashi, H. Ohki, Y. Shimizu, H. Okada and M. Tanimoto, Non-Abelian Discrete Symmetries in Particle Physics, Prog. Theor. Phys. Suppl. 183 (2010) 1 [arXiv: 1003.3552] [INSPIRE].

[31] R. de Adelhart Toorop, F. Bazzocchi, L. Merlo and A. Paris, Constraining Flavour Symmetries At The EW Scale I: The A A Higgs Potential, JHEP 03 (2011) 035 [Erratum ibid. 1301 (2013) 098] [arXiv:1012.1791] [INSPIRE]. 
[32] R. de Adelhart Toorop, F. Bazzocchi, L. Merlo and A. Paris, Constraining Flavour Symmetries At The EW Scale II: The Fermion Processes, JHEP 03 (2011) 040 [arXiv: 1012.2091] [INSPIRE].

[33] L. Merlo, S. Rigolin and B. Zaldivar, Flavour violation in a supersymmetric T' model, JHEP 11 (2011) 047 [arXiv:1108.1795] [INSPIRE].

[34] G. Altarelli, F. Feruglio, L. Merlo and E. Stamou, Discrete Flavour Groups, theta ${ }_{13}$ and Lepton Flavour Violation, JHEP 08 (2012) 021 [arXiv: 1205.4670] [INSPIRE].

[35] T2K collaboration, K. Abe et al., Indication of Electron Neutrino Appearance from an Accelerator-produced Off-axis Muon Neutrino Beam, Phys. Rev. Lett. 107 (2011) 041801 [arXiv:1106.2822] [INSPIRE].

[36] MINOS collaboration, P. Adamson et al., Improved search for muon-neutrino to electron-neutrino oscillations in MINOS, Phys. Rev. Lett. 107 (2011) 181802 [arXiv:1108.0015] [INSPIRE].

[37] Double CHOOZ collaboration, Y. Abe et al., Indication of Reactor $\bar{\nu}_{e}$ Disappearance in the Double CHOOZ Experiment, Phys. Rev. Lett. 108 (2012) 131801 [arXiv:1112.6353] [INSPIRE].

[38] Daya BAy collaboration, F.P. An et al., Observation of electron-antineutrino disappearance at Daya Bay, Phys. Rev. Lett. 108 (2012) 171803 [arXiv:1203.1669] [INSPIRE].

[39] RENO collaboration, J.K. Ahn et al., Observation of Reactor Electron Antineutrino Disappearance in the RENO Experiment, Phys. Rev. Lett. 108 (2012) 191802 [arXiv: 1204.0626] [INSPIRE].

[40] R.S. Chivukula and H. Georgi, Composite Technicolor Standard Model, Phys. Lett. B 188 (1987) 99 [INSPIRE].

[41] G. D'Ambrosio, G.F. Giudice, G. Isidori and A. Strumia, Minimal flavor violation: An Effective field theory approach, Nucl. Phys. B 645 (2002) 155 [hep-ph/0207036] [INSPIRE].

[42] V. Cirigliano, B. Grinstein, G. Isidori and M.B. Wise, Minimal flavor violation in the lepton sector, Nucl. Phys. B 728 (2005) 121 [hep-ph/0507001] [INSPIRE].

[43] S. Davidson and F. Palorini, Various definitions of Minimal Flavour Violation for Leptons, Phys. Lett. B 642 (2006) 72 [hep-ph/0607329] [INSPIRE].

[44] R. Alonso, G. Isidori, L. Merlo, L.A. Muñoz and E. Nardi, Minimal flavour violation extensions of the seesaw, JHEP 06 (2011) 037 [arXiv:1103.5461] [INSPIRE].

[45] D.N. Dinh, L. Merlo, S.T. Petcov and R. Vega-Álvarez, Revisiting Minimal Lepton Flavour Violation in the Light of Leptonic CP-violation, JHEP 07 (2017) 089 [arXiv:1705.09284] [INSPIRE].

[46] V. Cirigliano and B. Grinstein, Phenomenology of minimal lepton flavor violation, Nucl. Phys. B 752 (2006) 18 [hep-ph/0601111] [inSPIRE].

[47] B. Grinstein, V. Cirigliano, G. Isidori and M.B. Wise, Grand Unification and the Principle of Minimal Flavor Violation, Nucl. Phys. B 763 (2007) 35 [hep-ph/0608123] [INSPIRE].

[48] T. Hurth, G. Isidori, J.F. Kamenik and F. Mescia, Constraints on New Physics in MFV models: A Model-independent analysis of $\Delta F=1$ processes, Nucl. Phys. B 808 (2009) 326 [arXiv: 0807.5039] [INSPIRE].

[49] A.L. Kagan, G. Perez, T. Volansky and J. Zupan, General Minimal Flavor Violation, Phys. Rev. D 80 (2009) 076002 [arXiv:0903.1794] [INSPIRE]. 
[50] M.B. Gavela, T. Hambye, D. Hernandez and P. Hernández, Minimal Flavour Seesaw Models, JHEP 09 (2009) 038 [arXiv:0906.1461] [INSPIRE].

[51] B. Grinstein, M. Redi and G. Villadoro, Low Scale Flavor Gauge Symmetries, JHEP 11 (2010) 067 [arXiv: 1009.2049] [INSPIRE].

[52] T. Feldmann, See-Saw Masses for Quarks and Leptons in SU(5), JHEP 04 (2011) 043 [arXiv: 1010.2116] [INSPIRE].

[53] D. Guadagnoli, R.N. Mohapatra and I. Sung, Gauged Flavor Group with Left-Right Symmetry, JHEP 04 (2011) 093 [arXiv: 1103.4170] [INSPIRE].

[54] A.J. Buras, L. Merlo and E. Stamou, The Impact of Flavour Changing Neutral Gauge Bosons on $\bar{B} \rightarrow X_{s} \gamma$, JHEP 08 (2011) 124 [arXiv:1105.5146] [INSPIRE].

[55] A.J. Buras, M.V. Carlucci, L. Merlo and E. Stamou, Phenomenology of a Gauged SU(3) ${ }^{3}$ Flavour Model, JHEP 03 (2012) 088 [arXiv:1112.4477] [INSPIRE].

[56] R. Alonso, M.B. Gavela, L. Merlo, S. Rigolin and J. Yepes, Minimal Flavour Violation with Strong Higgs Dynamics, JHEP 06 (2012) 076 [arXiv:1201.1511] [INSPIRE].

[57] G. Isidori and D.M. Straub, Minimal Flavour Violation and Beyond, Eur. Phys. J. C 72 (2012) 2103 [arXiv: 1202.0464] [inSPIRE].

[58] L. Lopez-Honorez and L. Merlo, Dark matter within the minimal flavour violation ansatz, Phys. Lett. B 722 (2013) 135 [arXiv:1303.1087] [InSPIRE].

[59] F. Bishara, A. Greljo, J.F. Kamenik, E. Stamou and J. Zupan, Dark Matter and Gauged Flavor Symmetries, JHEP 12 (2015) 130 [arXiv:1505.03862] [INSPIRE].

[60] C.-J. Lee and J. Tandean, Minimal lepton flavor violation implications of the $b \rightarrow s$ anomalies, JHEP 08 (2015) 123 [arXiv: 1505. 04692] [INSPIRE].

[61] T. Feldmann, C. Luhn and P. Moch, Lepton-flavour violation in a Pati-Salam model with gauged flavour symmetry, JHEP 11 (2016) 078 [arXiv: 1608.04124] [INSPIRE].

[62] R. Alonso, E. Fernandez Martinez, M.B. Gavela, B. Grinstein, L. Merlo and P. Quilez, Gauged Lepton Flavour, JHEP 12 (2016) 119 [arXiv:1609.05902] [INSPIRE].

[63] G. Isidori, Y. Nir and G. Perez, Flavor Physics Constraints for Physics Beyond the Standard Model, Ann. Rev. Nucl. Part. Sci. 60 (2010) 355 [arXiv: 1002.0900] [InSPIRE].

[64] R. Alonso, M.B. Gavela, L. Merlo and S. Rigolin, On the scalar potential of minimal flavour violation, JHEP 07 (2011) 012 [arXiv: 1103.2915] [INSPIRE].

[65] R. Alonso, M.B. Gavela, D. Hernandez and L. Merlo, On the Potential of Leptonic Minimal Flavour Violation, Phys. Lett. B $\mathbf{7 1 5}$ (2012) 194 [arXiv:1206.3167] [INSPIRE].

[66] R. Alonso, M.B. Gavela, D. Hernández, L. Merlo and S. Rigolin, Leptonic Dynamical Yukawa Couplings, JHEP 08 (2013) 069 [arXiv:1306.5922] [INSPIRE].

[67] R. Alonso, M.B. Gavela, G. Isidori and L. Maiani, Neutrino Mixing and Masses from a Minimum Principle, JHEP 11 (2013) 187 [arXiv:1306.5927] [INSPIRE].

[68] A. Anselm and Z. Berezhiani, Weak mixing angles as dynamical degrees of freedom, Nucl. Phys. B 484 (1997) 97 [hep-ph/9605400] [inSPIRE].

[69] R. Barbieri, L.J. Hall, G.L. Kane and G.G. Ross, Nearly degenerate neutrinos and broken flavor symmetry, hep-ph/9901228 [INSPIRE].

[70] Z. Berezhiani and A. Rossi, Flavor structure, flavor symmetry and supersymmetry, Nucl. Phys. Proc. Suppl. 101 (2001) 410 [hep-ph/0107054] [INSPIRE]. 
[71] T. Feldmann, M. Jung and T. Mannel, Sequential Flavour Symmetry Breaking, Phys. Rev. D 80 (2009) 033003 [arXiv:0906.1523] [InSPIRE].

[72] E. Nardi, Naturally large Yukawa hierarchies, Phys. Rev. D 84 (2011) 036008 [arXiv:1105.1770] [INSPIRE].

[73] R.D. Peccei and H.R. Quinn, CP Conservation in the Presence of Instantons, Phys. Rev. Lett. 38 (1977) 1440 [InSPIRE].

[74] F. Wilczek, Problem of Strong $p$ and $t$ Invariance in the Presence of Instantons, Phys. Rev. Lett. 40 (1978) 279 [INSPIRE].

[75] S. Weinberg, A New Light Boson?, Phys. Rev. Lett. 40 (1978) 223 [InSPIRE].

[76] G. Grilli di Cortona, E. Hardy, J. Pardo Vega and G. Villadoro, The QCD axion, precisely, JHEP 01 (2016) 034 [arXiv: 1511.02867] [INSPIRE].

[77] J.E. Kim, Weak Interaction Singlet and Strong CP Invariance, Phys. Rev. Lett. 43 (1979) 103 [INSPIRE].

[78] M.A. Shifman, A.I. Vainshtein and V.I. Zakharov, Can Confinement Ensure Natural CP Invariance of Strong Interactions?, Nucl. Phys. B 166 (1980) 493 [INSPIRE].

[79] A.R. Zhitnitsky, On Possible Suppression of the Axion Hadron Interactions. (In Russian), Sov. J. Nucl. Phys. 31 (1980) 260 [inSPIRE].

[80] M. Dine, W. Fischler and M. Srednicki, A Simple Solution to the Strong CP Problem with a Harmless Axion, Phys. Lett. 104B (1981) 199 [InSPIRE].

[81] Y. Ema, K. Hamaguchi, T. Moroi and K. Nakayama, Flaxion: a minimal extension to solve puzzles in the standard model, JHEP 01 (2017) 096 [arXiv: 1612.05492] [INSPIRE].

[82] L. Calibbi, F. Goertz, D. Redigolo, R. Ziegler and J. Zupan, Minimal axion model from flavor, Phys. Rev. D 95 (2017) 095009 [arXiv: 1612.08040] [inSPIRE].

[83] F. Wilczek, Axions and Family Symmetry Breaking, Phys. Rev. Lett. 49 (1982) 1549 [INSPIRE].

[84] M.E. Albrecht, T. Feldmann and T. Mannel, Goldstone Bosons in Effective Theories with Spontaneously Broken Flavour Symmetry, JHEP 10 (2010) 089 [arXiv:1002.4798] [INSPIRE].

[85] S. Weinberg, Baryon and Lepton Nonconserving Processes, Phys. Rev. Lett. 43 (1979) 1566 [INSPIRE].

[86] K. Choi, S.H. Im, C.B. Park and S. Yun, Minimal Flavor Violation with Axion-like Particles, arXiv:1708.00021 [INSPIRE].

[87] D.B. Kaplan and H. Georgi, SU(2) $\times$ U(1) Breaking by Vacuum Misalignment, Phys. Lett. 136B (1984) 183 [inSPIRE].

[88] D.B. Kaplan, H. Georgi and S. Dimopoulos, Composite Higgs Scalars, Phys. Lett. 136B (1984) 187 [INSPIRE].

[89] T. Banks, Constraints on $\mathrm{SU}(2) \times \mathrm{U}(1)$ breaking by vacuum misalignment, Nucl. Phys. B 243 (1984) 125 [inSPIRE].

[90] K. Agashe, R. Contino and A. Pomarol, The Minimal composite Higgs model, Nucl. Phys. B 719 (2005) 165 [hep-ph/0412089] [INSPIRE].

[91] B. Gripaios, A. Pomarol, F. Riva and J. Serra, Beyond the Minimal Composite Higgs Model, JHEP 04 (2009) 070 [arXiv:0902.1483] [INSPIRE]. 
[92] J. Mrazek, A. Pomarol, R. Rattazzi, M. Redi, J. Serra and A. Wulzer, The Other Natural Two Higgs Doublet Model, Nucl. Phys. B 853 (2011) 1 [arXiv:1105.5403] [InSPIRE].

[93] R. Alonso, I. Brivio, B. Gavela, L. Merlo and S. Rigolin, Sigma Decomposition, JHEP 12 (2014) 034 [arXiv: 1409.1589] [INSPIRE].

[94] G. Panico and A. Wulzer, The Composite Nambu-Goldstone Higgs, Lect. Notes Phys. 913 (2016) pp.1-316 [arXiv:1506.01961] [INSPIRE].

[95] I.M. Hierro, L. Merlo and S. Rigolin, Sigma Decomposition: The CP-Odd Lagrangian, JHEP 04 (2016) 016 [arXiv:1510.07899] [INSPIRE].

[96] E. Halyo, Technidilaton or Higgs?, Mod. Phys. Lett. A 8 (1993) 275 [InSPIRE].

[97] W.D. Goldberger, B. Grinstein and W. Skiba, Distinguishing the Higgs boson from the dilaton at the Large Hadron Collider, Phys. Rev. Lett. 100 (2008) 111802 [arXiv: 0708.1463] [INSPIRE].

[98] L. Vecchi, Phenomenology of a light scalar: the dilaton, Phys. Rev. D 82 (2010) 076009 [arXiv: 1002.1721] [INSPIRE].

[99] S. Matsuzaki and K. Yamawaki, Is $125 \mathrm{GeV}$ techni-dilaton found at LHC?, Phys. Lett. B 719 (2013) 378 [arXiv:1207.5911] [INSPIRE].

[100] Z. Chacko and R.K. Mishra, Effective Theory of a Light Dilaton, Phys. Rev. D 87 (2013) 115006 [arXiv:1209.3022] [INSPIRE].

[101] Z. Chacko, R. Franceschini and R.K. Mishra, Resonance at $125 \mathrm{GeV}$ : Higgs or Dilaton/Radion?, JHEP 04 (2013) 015 [arXiv: 1209.3259] [INSPIRE].

[102] B. Bellazzini, C. Csáki, J. Hubisz, J. Serra and J. Terning, A Higgslike Dilaton, Eur. Phys. J. C 73 (2013) 2333 [arXiv: 1209.3299] [INSPIRE].

[103] P. Hernández-Leon and L. Merlo, Distinguishing A Higgs-Like Dilaton Scenario With A Complete Bosonic Effective Field Theory Basis, Phys. Rev. D 96 (2017) 075008 [arXiv: 1703. 02064] [INSPIRE].

[104] F. Feruglio, The Chiral approach to the electroweak interactions, Int. J. Mod. Phys. A 8 (1993) 4937 [hep-ph/9301281] [INSPIRE].

[105] B. Grinstein and M. Trott, A Higgs-Higgs bound state due to new physics at a TeV, Phys. Rev. D 76 (2007) 073002 [arXiv:0704.1505] [InSPIRE].

[106] R. Contino, C. Grojean, M. Moretti, F. Piccinini and R. Rattazzi, Strong Double Higgs Production at the LHC, JHEP 05 (2010) 089 [arXiv:1002.1011] [INSPIRE].

[107] R. Alonso, M.B. Gavela, L. Merlo, S. Rigolin and J. Yepes, The Effective Chiral Lagrangian for a Light Dynamical "Higgs Particle", Phys. Lett. B 722 (2013) 330 [Erratum ibid. B 726 (2013) 926] [arXiv: 1212.3305] [INSPIRE].

[108] R. Alonso, M.B. Gavela, L. Merlo, S. Rigolin and J. Yepes, Flavor with a light dynamical "Higgs particle", Phys. Rev. D 87 (2013) 055019 [arXiv:1212.3307] [INSPIRE].

[109] G. Buchalla, O. Catà and C. Krause, Complete Electroweak Chiral Lagrangian with a Light Higgs at NLO, Nucl. Phys. B 880 (2014) 552 [Erratum ibid. B 913 (2016) 475] [arXiv: 1307.5017$]$ [INSPIRE].

[110] I. Brivio et al., Disentangling a dynamical Higgs, JHEP 03 (2014) 024 [arXiv:1311.1823] [INSPIRE]. 
[111] I. Brivio, O.J.P. É boli, M.B. Gavela, M.C. Gonzalez-Garcia, L. Merlo and S. Rigolin, Higgs ultraviolet softening, JHEP 12 (2014) 004 [arXiv: 1405.5412] [INSPIRE].

[112] M.B. Gavela, J. Gonzalez-Fraile, M.C. Gonzalez-Garcia, L. Merlo, S. Rigolin and J. Yepes, CP violation with a dynamical Higgs, JHEP 10 (2014) 044 [arXiv: 1406.6367] [INSPIRE].

[113] M.B. Gavela, K. Kanshin, P.A.N. Machado and S. Saa, On the renormalization of the electroweak chiral Lagrangian with a Higgs, JHEP 03 (2015) 043 [arXiv:1409.1571] [INSPIRE].

[114] I. Brivio et al., Non-linear Higgs portal to Dark Matter, JHEP 04 (2016) 141 [arXiv: 1511.01099] [INSPIRE].

[115] B.M. Gavela, E.E. Jenkins, A.V. Manohar and L. Merlo, Analysis of General Power Counting Rules in Effective Field Theory, Eur. Phys. J. C 76 (2016) 485 [arXiv: 1601.07551] [INSPIRE].

[116] R. Alonso, E.E. Jenkins and A.V. Manohar, $\sigma$-models with Negative Curvature, Phys. Lett. B 756 (2016) 358 [arXiv: 1602.00706] [InSPIRE].

[117] O.J.P. É boli and M.C. Gonzalez-Garcia, Classifying the bosonic quartic couplings, Phys. Rev. D 93 (2016) 093013 [arXiv: 1604.03555] [InSPIRE].

[118] I. Brivio, J. Gonzalez-Fraile, M.C. Gonzalez-Garcia and L. Merlo, The complete HEFT Lagrangian after the LHC Run I, Eur. Phys. J. C 76 (2016) 416 [arXiv:1604.06801] [INSPIRE].

[119] R. Alonso, E.E. Jenkins and A.V. Manohar, Geometry of the Scalar Sector, JHEP 08 (2016) 101 [arXiv:1605.03602] [INSPIRE].

[120] LHC Higgs Cross Section Working Group collaboration, Handbook of LHC Higgs Cross Sections: 4. Deciphering the Nature of the Higgs Sector, arXiv:1610.07922 [INSPIRE].

[121] L. Merlo, S. Saa and M. Sacristán-Barbero, Baryon Non-Invariant Couplings in Higgs Effective Field Theory, Eur. Phys. J. C 77 (2017) 185 [arXiv: 1612.04832] [InSPIRE].

[122] G.F. Giudice, R. Rattazzi and A. Strumia, Unificaxion, Phys. Lett. B 715 (2012) 142 [arXiv: 1204.5465] [INSPIRE].

[123] M. Redi and A. Strumia, Axion-Higgs Unification, JHEP 11 (2012) 103 [arXiv:1208.6013] [INSPIRE].

[124] M. Redi and R. Sato, Composite Accidental Axions, JHEP 05 (2016) 104 [arXiv: 1602 .05427] [INSPIRE].

[125] L. Di Luzio, F. Mescia and E. Nardi, Redefining the Axion Window, Phys. Rev. Lett. 118 (2017) 031801 [arXiv:1610.07593] [INSPIRE].

[126] M. Farina, D. Pappadopulo, F. Rompineve and A. Tesi, The photo-philic QCD axion, JHEP 01 (2017) 095 [arXiv: 1611.09855] [INSPIRE].

[127] R. Coy, M. Frigerio and M. Ibe, Dynamical Clockwork Axions, JHEP 10 (2017) 002 [arXiv: 1706. 04529] [INSPIRE].

[128] K. Choi, K. Kang and J.E. Kim, Effects of $\eta^{\prime}$ in Low-energy Axion Physics, Phys. Lett. B 181 (1986) 145 [INSPIRE].

[129] J.D. Bjorken et al., Search for Neutral Metastable Penetrating Particles Produced in the SLAC Beam Dump, Phys. Rev. D 38 (1988) 3375 [inSPIRE]. 
[130] M. Carena and R.D. Peccei, The Effective Lagrangian for Axion Emission From SN1987A, Phys. Rev. D 40 (1989) 652 [InSPIRE].

[131] G.G. Raffelt, Astrophysical axion bounds, Lect. Notes Phys. 741 (2008) 51 [hep-ph/0611350] [INSPIRE].

[132] E787 and E949 collaborations, S. Adler et al., Measurement of the $K^{+} \rightarrow \pi^{+} \nu \bar{\nu}$ branching ratio, Phys. Rev. D 77 (2008) 052003 [arXiv: 0709.1000] [INSPIRE].

[133] Borexino collaboration, G. Bellini et al., Search for Solar Axions Produced in $p\left(d,{ }^{3} \mathrm{He}\right) \mathrm{A}$ Reaction with Borexino Detector, Phys. Rev. D 85 (2012) 092003 [arXiv: 1203.6258] [INSPIRE].

[134] A. Friedland, M. Giannotti and M. Wise, Constraining the Axion-Photon Coupling with Massive Stars, Phys. Rev. Lett. 110 (2013) 061101 [arXiv:1210.1271] [InSPIRE].

[135] BABAR collaboration, J.P. Lees et al., Search for $B \rightarrow K^{(*)} \nu \bar{\nu}$ and invisible quarkonium decays, Phys. Rev. D 87 (2013) 112005 [arXiv: 1303.7465] [inSPIRE].

[136] E. Armengaud et al., Axion searches with the EDELWEISS-II experiment, JCAP 11 (2013) 067 [arXiv: 1307.1488] [INSPIRE].

[137] J.D. Clarke, R. Foot and R.R. Volkas, Phenomenology of a very light scalar $\left(100 \mathrm{MeV}<m_{h}<10 \mathrm{GeV}\right)$ mixing with the SM Higgs, JHEP 02 (2014) 123 [arXiv: 1310.8042] [INSPIRE].

[138] N. Viaux et al., Neutrino and axion bounds from the globular cluster M5 (NGC 5904), Phys. Rev. Lett. 111 (2013) 231301 [arXiv:1311.1669] [INSPIRE].

[139] XENON100 collaboration, E. Aprile et al., First Axion Results from the XENON100 Experiment, Phys. Rev. D 90 (2014) 062009 [arXiv: 1404.1455] [INSPIRE].

[140] A. Ayala, I. Domínguez, M. Giannotti, A. Mirizzi and O. Straniero, Revisiting the bound on axion-photon coupling from Globular Clusters, Phys. Rev. Lett. 113 (2014) 191302 [arXiv: 1406.6053] [INSPIRE].

[141] CMS collaboration, Search for dark matter, extra dimensions and unparticles in monojet events in proton-proton collisions at $\sqrt{s}=8 \mathrm{TeV}$, Eur. Phys. J. C $7 \mathbf{5}$ (2015) 235 [arXiv: 1408.3583] [INSPIRE].

[142] K. Mimasu and V. Sanz, ALPs at Colliders, JHEP 06 (2015) 173 [arXiv:1409.4792] [INSPIRE].

[143] M.J. Dolan, F. Kahlhoefer, C. McCabe and K. Schmidt-Hoberg, A taste of dark matter: Flavour constraints on pseudoscalar mediators, JHEP 03 (2015) 171 [Erratum ibid. 1507 (2015) 103] [arXiv: 1412.5174] [INSPIRE].

[144] M. Millea, L. Knox and B. Fields, New Bounds for Axions and Axion-Like Particles with keV-GeV Masses, Phys. Rev. D 92 (2015) 023010 [arXiv:1501.04097] [InSPIRE].

[145] N. Vinyoles, A. Serenelli, F.L. Villante, S. Basu, J. Redondo and J. Isern, New axion and hidden photon constraints from a solar data global fit, JCAP 10 (2015) 015 [arXiv: 1501.01639] [INSPIRE].

[146] ATLAS collaboration, Search for new phenomena in final states with an energetic jet and large missing transverse momentum in pp collisions at $\sqrt{s}=8 \mathrm{TeV}$ with the ATLAS detector, Eur. Phys. J. C 75 (2015) 299 [arXiv:1502.01518] [INSPIRE].

[147] G. Krnjaic, Probing Light Thermal Dark-Matter With a Higgs Portal Mediator, Phys. Rev. D 94 (2016) 073009 [arXiv:1512.04119] [InSPIRE]. 
[148] W.J. Marciano, A. Masiero, P. Paradisi and M. Passera, Contributions of axionlike particles to lepton dipole moments, Phys. Rev. D 94 (2016) 115033 [arXiv:1607.01022] [INSPIRE].

[149] E. Izaguirre, T. Lin and B. Shuve, Searching for Axionlike Particles in Flavor-Changing Neutral Current Processes, Phys. Rev. Lett. 118 (2017) 111802 [arXiv:1611.09355] [INSPIRE].

[150] I. Brivio et al., ALPs Effective Field Theory and Collider Signatures, Eur. Phys. J. C 77 (2017) 572 [arXiv:1701.05379] [INSPIRE].

[151] M. Bauer, M. Neubert and A. Thamm, LHC as an Axion Factory: Probing an Axion Explanation for $(g-2)_{\mu}$ with Exotic Higgs Decays, Phys. Rev. Lett. 119 (2017) 031802 [arXiv: 1704.08207] [INSPIRE].

[152] CAST collaboration, V. Anastassopoulos et al., New CAST Limit on the Axion-Photon Interaction, Nature Phys. 13 (2017) 584 [arXiv:1705.02290] [INSPIRE].

[153] M.J. Dolan, T. Ferber, C. Hearty, F. Kahlhoefer and K. Schmidt-Hoberg, Revised constraints and Belle II sensitivity for visible and invisible axion-like particles, arXiv:1709.00009 [INSPIRE].

[154] J. Jaeckel and M. Spannowsky, Probing MeV to $90 \mathrm{GeV}$ axion-like particles with LEP and LHC, Phys. Lett. B 753 (2016) 482 [arXiv:1509.00476] [INSPIRE].

[155] M. Bauer, M. Neubert and A. Thamm, Collider Probes of Axion-Like Particles, arXiv: 1708.00443 [INSPIRE].

[156] W.A. Bardeen, S.H.H. Tye and J.A.M. Vermaseren, Phenomenology of the new light Higgs boson search, Phys. Lett. B 76 (1978) 580.

[157] P. Di Vecchia and G. Veneziano, Chiral Dynamics in the Large-N Limit, Nucl. Phys. B 171 (1980) 253 [inSPIRE].

[158] S.M. Barr and D. Seckel, Planck scale corrections to axion models, Phys. Rev. D 46 (1992) 539 [inSPIRE].

[159] M. Kamionkowski and J. March-Russell, Planck scale physics and the Peccei-Quinn mechanism, Phys. Lett. B 282 (1992) 137 [hep-th/9202003] [INSPIRE].

[160] R. Holman, S.D.H. Hsu, T.W. Kephart, E.W. Kolb, R. Watkins and L.M. Widrow, Solutions to the strong CP problem in a world with gravity, Phys. Lett. B 282 (1992) 132 [hep-ph/9203206] [INSPIRE].

[161] R. Alonso and A. Urbano, Wormholes and masses for Goldstone bosons, arXiv: 1706.07415 [INSPIRE].

[162] F. Arias-Aragon, L. Merlo and E. Stamou, work in progress.

[163] S. Cunliffe, Prospects for rare B decays at Belle II, arXiv:1708.09423 [INSPIRE]. 